\title{
Performance Evaluation of Low-Cost and Real-Time Multi- GNSS Advanced Demonstration Tool for Orbit and Clock Analysis-Precise Point Positioning (MADOCA-PPP) Receiver Systems
}

\author{
Ellarizza Fredeluces ${ }^{1 *}$, Arthur Rey Fabian Lagura ${ }^{1}$, Rosalie Reyes ${ }^{1}$, Nobuaki Kubo ${ }^{2}$ \\ ${ }^{1}$ Department of Geodetic Engineering, University of the Philipines \\ Diliman, Quezon City, Philippines \\ 2 Tokyo University Marine Science and Technology \\ Tokyo, Japan \\ *Corresponding author's email: etfredeluces [AT] up.edu.ph
}

\begin{abstract}
With Multi-GNSS Advanced Demonstration Tool for Orbit and Clock Analysis (MADOCA), a software estimator of precise satellite information, by JAXA, u-blox C099 ZED-F9P and MSJ-3008-GM4-QZS using MADOCA-PPP can be exploited in GNSS applications that require sub-decimeter accuracy without being costly. To evaluate their performance, convergence time and accuracy of solutions are compared to Trimble NetR9, a survey-grade receiver. Post-processed PPP solutions of ZED-F9P were computed using RTKLIB and real-time $P P P$ was provided by the MSJ-3008-GM4-QZS. Results showed ZED-F9P achieved an RMS of $5.28 \mathrm{~cm}, 2.89 \mathrm{~cm}$, and $9.55 \mathrm{~cm}$ in East, North, and Up directions. This means ZED-F9P can be used in applications requiring below $10 \mathrm{~cm}$ accuracy even without base station. MSJ-3008-GM4-QZS obtained an RMS of $10.45 \mathrm{~cm}, 6.27 \mathrm{~cm}$, and 27.56 cm in the same directions. Unlike ZED-F9P, it achieved above $10 \mathrm{~cm}$ accuracy in North and Up directions which is due to large errors from cycle slips and jumps in observations. Interestingly, no fixed solutions were achieved between the 20:00 to 21:00 UTC period, although said receiver was able to receive corrections. However, if the observations after 20:00 UTC were removed, the MSJ-3008-GM4-QZS improved to $7.17 \mathrm{~cm}, 3.58 \mathrm{~cm}$, and 22.32 cm in the same directions.
\end{abstract}

Keywords- MADOCA-PPP, GNSS, RTKLIB

\section{INTRODUCTION}

The Quasi-Zenith Satellite System (QZSS) is a regional satellite positioning and timing system that mainly focuses on Japan and covers neighboring countries in Asia. QZSS navigation signals are designed to be similar with GPS; thus, their compatibility and interoperability with each other. These are L1-C/A, L1C, L2C, and L5 signals. It also has an augmentation signal, namely, L1-SAIF (Sub-meter class Augmentation with Integrity Function), that operates with satellite-based augmentation system (SBAS) through wide-area differential GPS (WDGPS) [1-3]. By providing GNSS interoperable and augmentation signals from its satellites placed in an inclined geostationary and quasi-zenith orbit, QZSS enables users in the region to have fair and consistent positioning even in urban and mountainous areas where signals are most likely to be hampered [4-7]. QZSS also broadcasts L-Band Experimental (LEX) signal. It has the same frequency as that of the Galileo E6 signal and delivers correction which includes precise satellite orbit and clock information, user-range accuracy (URA), and GNSS code and phase biases [2,8]. The LEX signal also includes a satellite message calculated by Multi-GNSS Advanced Demonstration Tool for Orbit and Clock Analysis (MADOCA). It is a software, developed by JAXA, that calculates precise satellite orbit and clock bias of present GNSS systems by utilizing the observation from GNSS monitoring stations distributed around the world [9].

MADOCA-LEX's precise point positioning (PPP) performance has been evaluated in different countries such as Australia [10], New Zealand [11], Philippines [12], and Vietnam [13], and tested in different precise point positioning application such as robotic [14], transportation [12], and precise farming [15], In these studies, utilization of said technology had been exploited. The demand for low-cost receivers that can achieve high precision and accuracy continues to rise. The achieved accuracy of said receivers are now comparable with survey-grade receivers albeit with limitations. Some PPP and GNSS studies have already tested single-frequency [12, 16-17], dual-frequency [18], and triple-frequency [19] low-cost receivers that usually utilizes the GPS system and their results showed encouraging results. In this study, MADOCA-LEX was tested in a low-cost receiver, u-blox C099 ZED-F9P, and compared to the 
performance of survey-grade receivers in a post-processed solution.

Some studies [13,20] have developed an algorithm to decode the MADOCA-LEX message due to the unavailability of receivers that can automatically process said signal during that time of the experimentations. Nowadays, there are new receivers that support QZSS and decode the LEX signal to use the correction information [21]. One of these is the MSJ-3008-GM 4-QZS developed by Magellan Systems Japan, Inc. This study aims to evaluate its PPP performance in real time by comparing it to the Trimble NetR9 receiver post-process solution. Additionally, the evaluation of MSJ-3008-GM 4-QZS receiver is also being conducted in some countries in the East Asia and Oceania Region [22]. This study also tests the MADOCA-LEX signal availability in the Philippines. An initial test related to this was conducted by Reyes et. $\mathrm{Al}[12]$.

Low-cost receivers using MADOCA-PPP can be a potential substitute to applications that use survey gradereceivers and base stations. These can also achieve centimeter-class accuracy with less operational costs. With the recently developed receiver by Magellan Systems Japan, Inc., external devices, and algorithms to decode the LEX signal in real-time mode can be eliminated. This study limits only its application in static observations. Also, the observations were only done in an open-sky environment and both receivers were simultaneously taking observations using only one antenna. GPS, GLONASS, and QZSS are the only satellite systems that were used because MADOCA, as of this writing, can only be applied in these three systems [24]. The accuracy of the solutions was evaluated against the true position of the Trimble NetR9 base station.

\section{METHODOLOGY}

The general method for this study consisted of three parts, namely: (1) data gathering, (2) data processing, and (3) data analysis. The workflow of the procedure is illustrated in Figure 1.

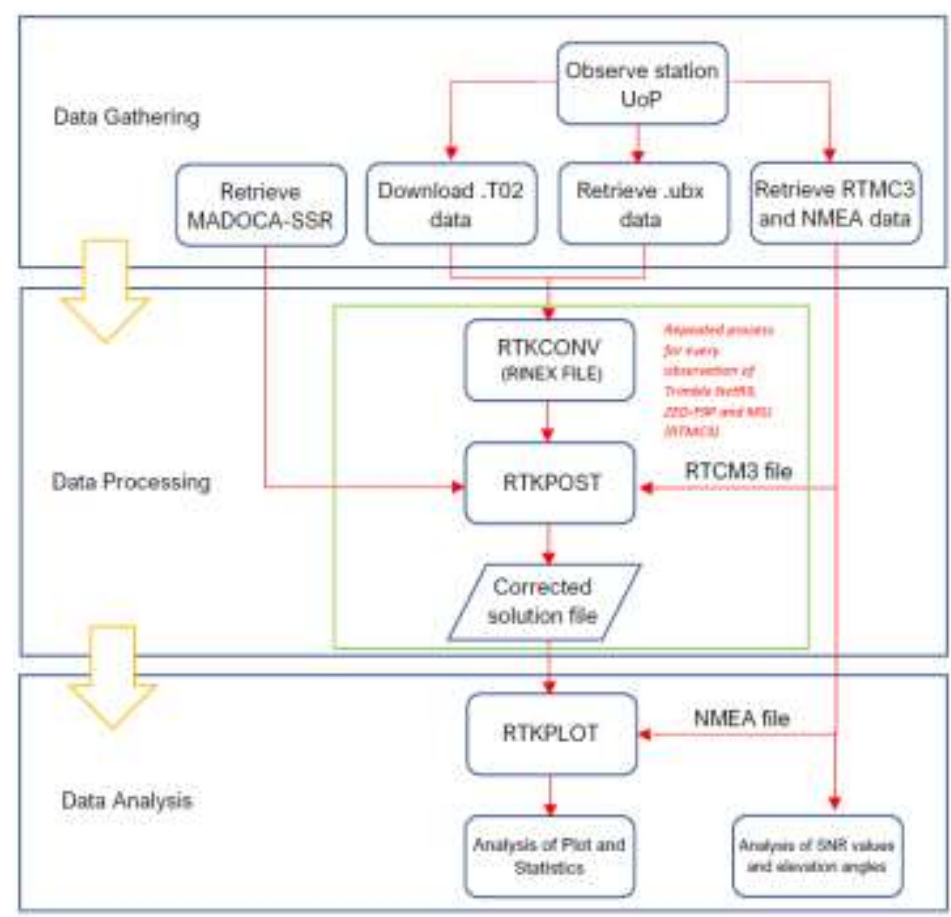

Figure 1: General Workflow of the study

\subsection{Study Area}

The active base station UoP was used as a test site. This station is equipped with a Trimble NetR9 receiver with antenna located at the rooftop of College of Engineering in University of the Philippines, Diliman campus. Since its installation in 2015, the said receiver continuously collects GNSS signals. Its WGS84 coordinates were obtained using Trimble CenterPoint RTX Post-Processing Service and are indicated in Table 1. Tests for MADOCA-PPP applicability in post-processed static observation were conducted for the months of February and March 2020.

Table 1: WGS84 Coordinates of Active Geodetic Station

\begin{tabular}{cc}
\hline Latitude & $14^{\circ} 39^{\prime} 23.135^{\prime \prime} \mathrm{N}$ \\
Longitude & $121^{\circ} 04^{\prime} 11.117^{\prime \prime} \mathrm{E}$ \\
Ellipsoidal Height & $134.266 \mathrm{~m}$ \\
\hline
\end{tabular}




\subsection{Data Gathering}

GNSS signals were collected using the geodetic antenna Trimble Zephyr 3, mounted on the station, and transferred to three receivers that were connected to the antenna through a GNSS splitter. One of these, MSJ-3008-GM4-QZS, is under evaluation for PPP performance in different countries in the East and Oceania Region [22]. The u-blox ZEDF9P [24] is a low-cost receiver that supports multi-frequency and multi-GNSS observations; however, it can only receive limited GNSS signals. MSJ-3008-GM4-QZS is also a multi-frequency and multi-GNSS receiver designed by Magellan Systems Japan Inc., that can receive and decode L6 signal in real-time to achieve better accuracy than single point positioning [25]. The reference receiver to validate the performance of the two receivers being evaluated is the Trimble NetR9. The 24-hour observations were done every day for the whole month of February and March. However, due to technical interference, some days had incomplete or no data for the u-blox receiver. Corresponding MADOCA products for each available observation were retrieved from the JAXA website [26].

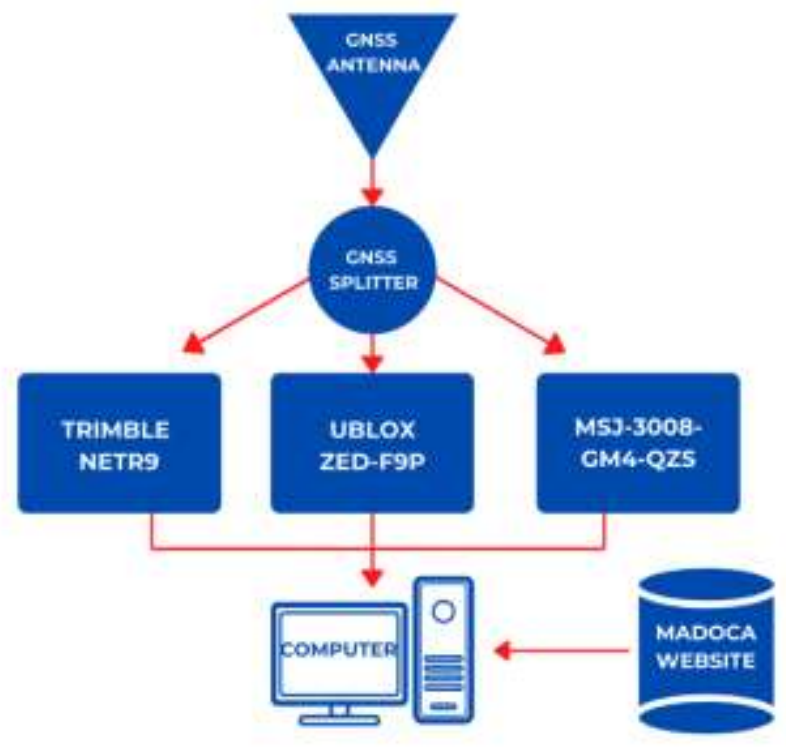

Figure 2: Data Gathering Setup

\subsection{Data Processing}

The RTKLIB [27], a software package developed by Tomoji Takasu, was used for the post-processing. The .ubx files were converted to RINEX files using RTKCONV while the .T02 data used the built-in conversion in the Trimble receiver. The converted files and the MADOCA correction products were the input for processing in RTKPOST. To get a solution at every epoch in the observation, the selected positioning mode in the settings was PPP. Only GPS, GLONASS and QZSS data were selected because MADOCA only works for this satellite system as of this writing. This process was repeated for all available observations for u-blox ZED-F9P and Trimble Net-R9. The NMEA file from MSJ-3008-GM4-QZS did not undergo post-processing since its output was already the corrected positions in real time.

\subsection{Data Analysis}

To examine how long u-blox ZED-F9P and Trimble NetR9 resolve the receiver biases or their solutions converge, the errors for the first 60 minutes of each observation were collected. Each solution file from u-blox ZED-F9P and MSJ-3008-GM4-QZS was compared to the solution of Trimble NetR9. The first 60 minutes in u-blox ZED-F9P and Trimble NetR9 solutions were filtered to exclude the observations during the convergence period. The average, standard deviation, and root mean square in East, North, and Up directions were generated by RTKPLOT with respect to the WGS84 coordinates of the station.

To check the availability of MADOCA-LEX in the Philippines, a program was developed by Tokyo University Marine Science and Technology (TUMSAT) Laboratory in Japan to extract the LEX signal and its relevant information, i.e. signal to noise ratio and elevation angles of QZSS from the NMEA file output of MSJ-3008-GM4QZS. 


\section{RESULTS AND DISCUSSIONS}

\subsection{Convergence analysis}

The convergence time is defined as no single epoch of positioning errors exceeding the threshold; however, there are some dates that have exceeded the $10 \mathrm{~cm}$ threshold after converging, but it eventually returned within the threshold. With errors oscillating around the threshold with very small amplitude, the convergence time can be less than the tabulated values. Table 2 shows the summary of the time of convergence for all observations of u-blox ZED-F9P and Trimble NetR9. The values show that both receivers converge almost at the same rate and less than 30 minutes. However, there is no recorded time in the Up direction that is less than $10 \mathrm{~cm}$ since this component usually is less accurate than the horizontal components. Trimble NetR9 has a faster convergence time in all 3 directions as compared to u-blox ZED-F9P. The difference is around 8 minutes and 5 seconds with ZED-F9P behind Trimble NetR9. Figures 3 and 4 illustrate the behavior of horizontal and vertical convergence for both receivers per minute during the first hour of observation. Trimble NetR9 has a more uniform convergence than ZED-F9P.

Table 2: Summary of convergence time

\begin{tabular}{ccc}
\hline Direction (threshold) & Trimble NetR9 & Ublox ZED-F9P \\
\hline E-W $(<10 \mathrm{~cm})$ & $0: 18: 28$ & $0: 25: 31$ \\
N-S $(<10 \mathrm{~cm})$ & $0: 15: 34$ & $0: 16: 49$ \\
U-D $(<20 \mathrm{~cm})$ & $0: 17: 54$ & $0: 26: 33$ \\
\hline
\end{tabular}

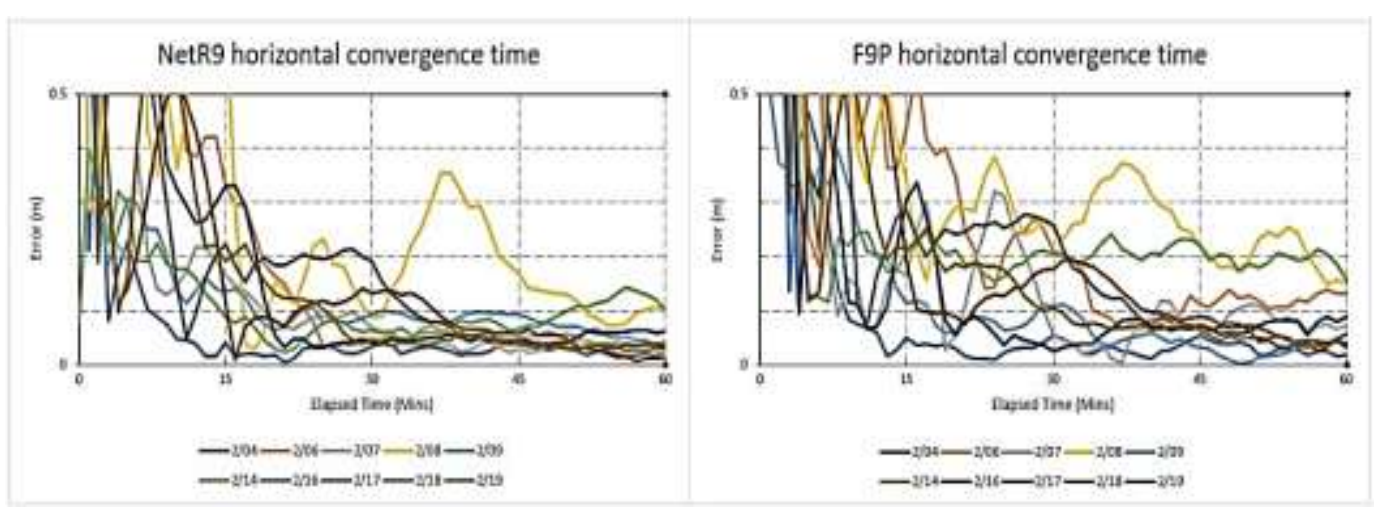

Figure 3: Trimble NetR9 and ZED-F9P horizontal convergence time

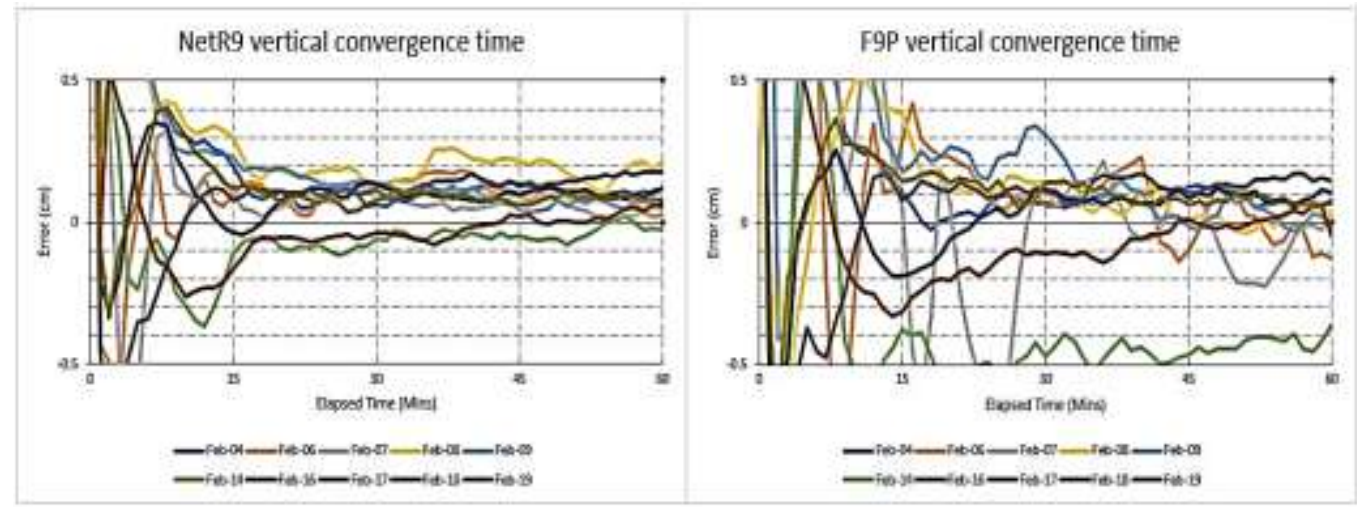

Figure 4: Trimble NetR9 and ZED-F9P vertical convergence time

The difference in convergence time can be primarily attributed to the hardware designs of the receivers. Since ZED-F9P is a low-cost receiver, it can only support limited GNSS signals, whereas Trimble NetR9, a survey-grade receiver, can utilize more signals since it is intended for high-accuracy applications. In addition, the North direction has the fastest convergence time for both receivers. This trend is attributed to the direction of motion of the observed satellites. Most of them are moving in the north to south direction as seen in Figure 5. 


\subsection{Comparison of U-blox ZED-F9P and Trimble NetR9}

To compare the accuracy of both receivers, the average, standard deviation, and root mean square for each day were recorded from RTKPLOT after the first hour of the observation is removed to eliminate the convergence period. Table 3 shows the average statistics of ZED-F9P and Trimble NetR9 in three directions for the whole month of February.

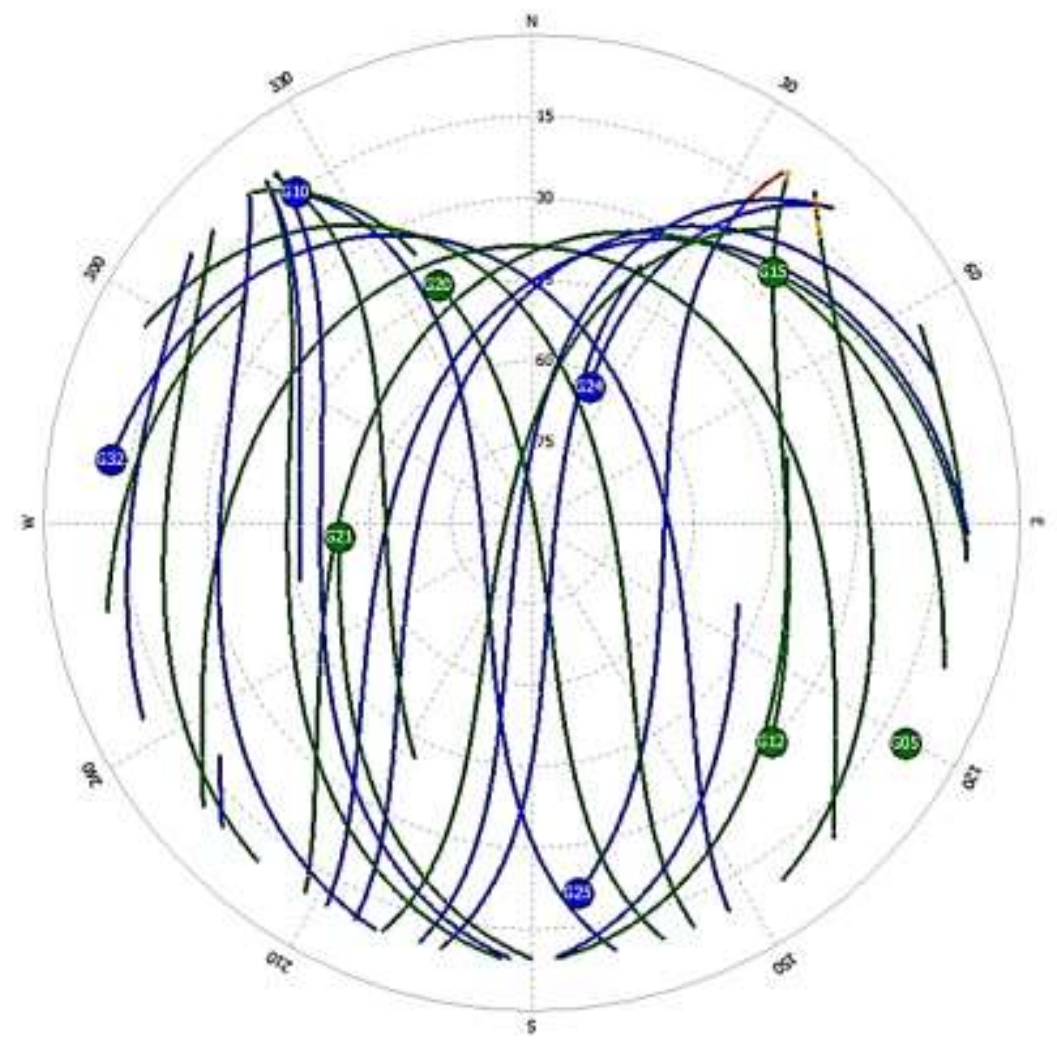

Figure 5: Direction of observed satellites

Table 3: ZED-F9P and Trimble NetR9 statistics (in $\mathrm{cm}$ )

\begin{tabular}{|c|ccc|ccc|}
\hline Direction & \multicolumn{3}{|c|}{ ZED-F9P } & \multicolumn{3}{c|}{ NetR9 } \\
\hline & Ave. & SD & RMS & Ave. & SD & RMS \\
E-W & 0.60 & 4.66 & 5.28 & 0.64 & 3.25 & 3.54 \\
N-S & 1.24 & 2.41 & 2.89 & 1.38 & 2.01 & 2.55 \\
U-D & 4.07 & 7.42 & 9.55 & 6.52 & 5.66 & 8.74 \\
\hline
\end{tabular}

Figures 6 and 7 show the comparison between the two receivers' statistics in the horizontal and vertical direction. Majority of recorded values are within centimeter level, except for ZED-F9P February 6 data with values of $21.29 \mathrm{~cm}$ and $21.85 \mathrm{~cm}$ for standard deviation and RMS, respectively, on the F9P east direction are the data obtained on February 6. This is because of the two cycle slips that happened during said data, and with each cycle slip, the observation had to re-converge. All other dates have favorable results with F9P able to achieve sub-centimeter mean and 1-2 cm standard deviation and RMS. From the same figures, the horizontal and vertical statistics of NetR9 and F9P are relatively the same. 


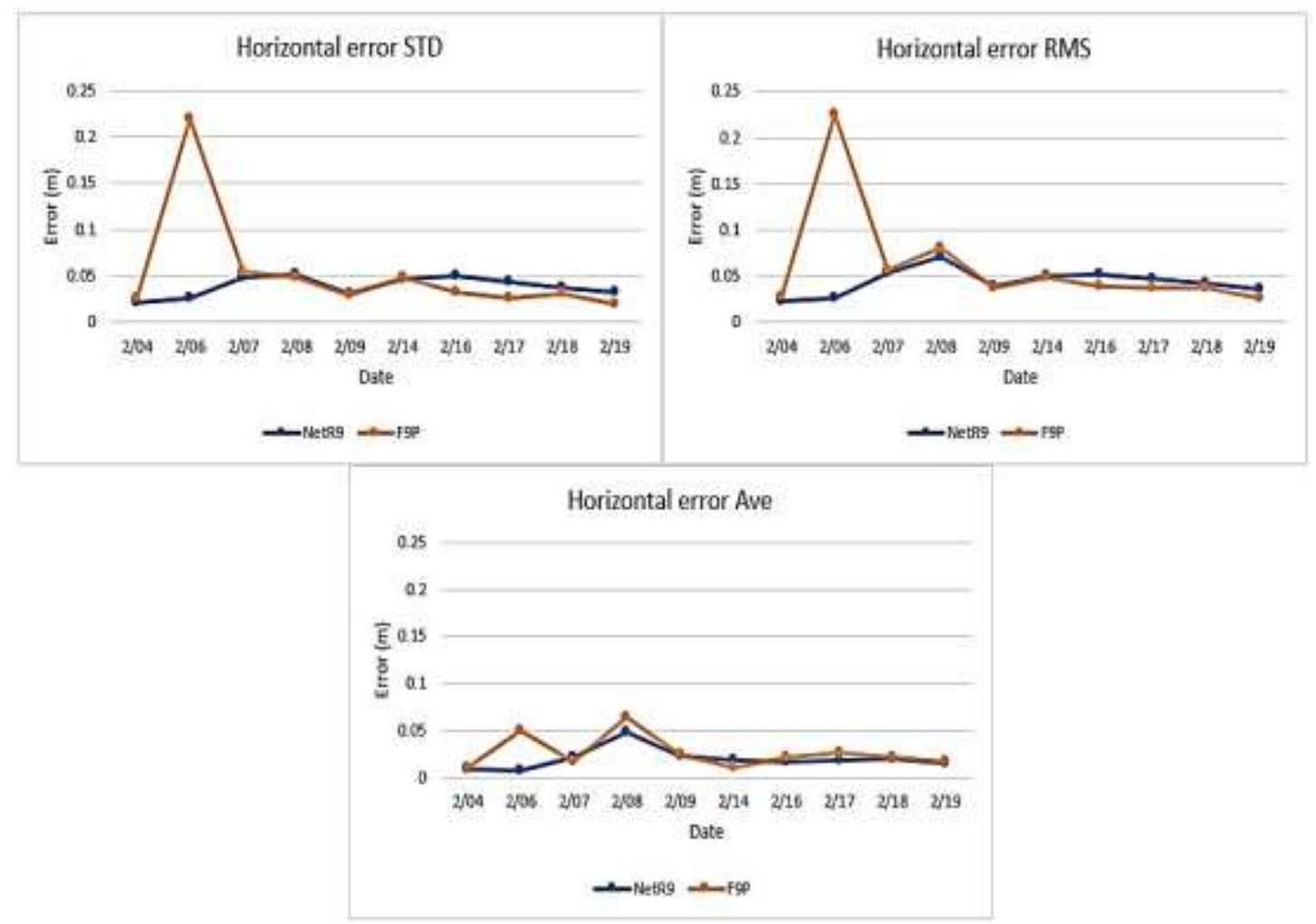

Figure 6: Horizontal error statistics plot

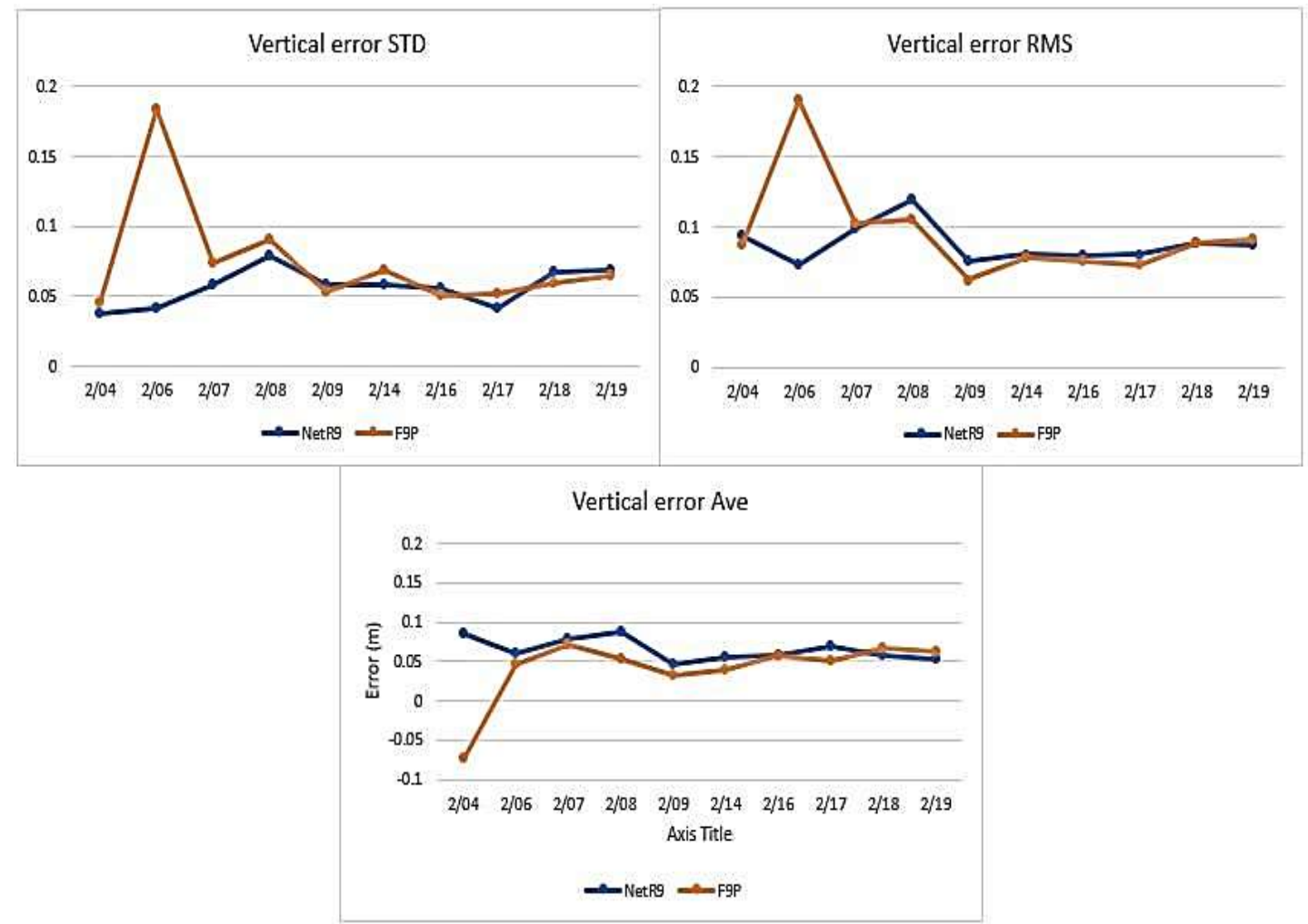

Figure 7: Vertical error statistics plot

\subsection{Availability of LEX Signal}

Figure 8a presents the visibility of QZSS satellites during the 24-hour observation on March 1, 2020. This shows that at every epoch, the receiver can receive all four QZSS satellites that broadcast the LEX signal at any time of the 
day. The high elevation of the satellites results in a relatively good signal reception of the LEX signal. Figure $8 \mathrm{~b}$ displays the signal to noise ratio values (SNR) at the first 10 minutes of the observation on March 1, 2020. At high elevation angles, the SNR values have an average of $50 \mathrm{~dB}$ and as the elevation angles decrease, the SNR values are also affected. The J03 satellite has a relatively lower SNR value than the other three because it was still rising from the horizon at this epoch.

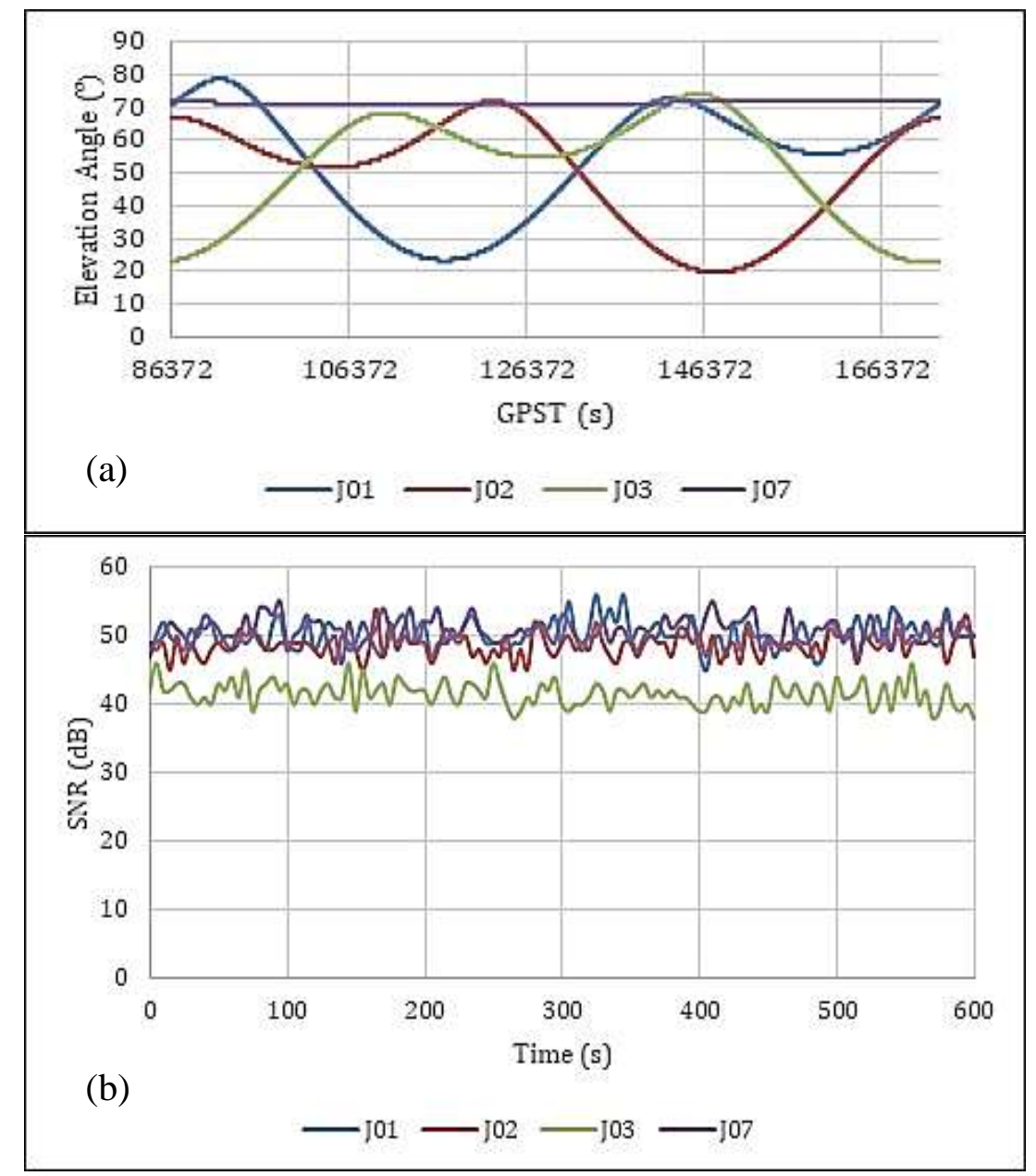

Figure 8: a) Elevation Angle of QZSS Satellites in UP Diliman (03/01/2020) and b) Signal to Noise Ratio of LEX signal during first 10 minutes

\subsection{Comparison of MSJ-3008-GM4-QZS and Trimble NetR9}

The plot of real-time solution of MSJ-3008-GM4-QZS on March 1, 2020 is shown in Figure 9a. It should be noted that there is no PPP solution between the 20:00 to 21:00 UTC period. This is displayed in RTKPLOT with Q $=5$ equivalent to a Single Point Solution. This means that the receiver is only calculating positions using code-based observations from the satellites. This also happened in some dates in February, on March 24 and March 26, 2020, where there are also no solutions in some periods. To check if it is a MADOCA system or receiver problem, the realtime solution is compared to the post-processed RTCM3 raw observation file. As presented in Figure 9b, there is a PPP solution in the period 20:00 to 21:00 UTC, which means that the receiver is receiving LEX signal at this period. This needs further verification with the developer of MSJ-3008-GM4-QZS to determine if this may be due to the receiver itself. As shown in Figure 10, the same problem was also noticed with the data from the same receiver installed in Australia for evaluation. 
(a)

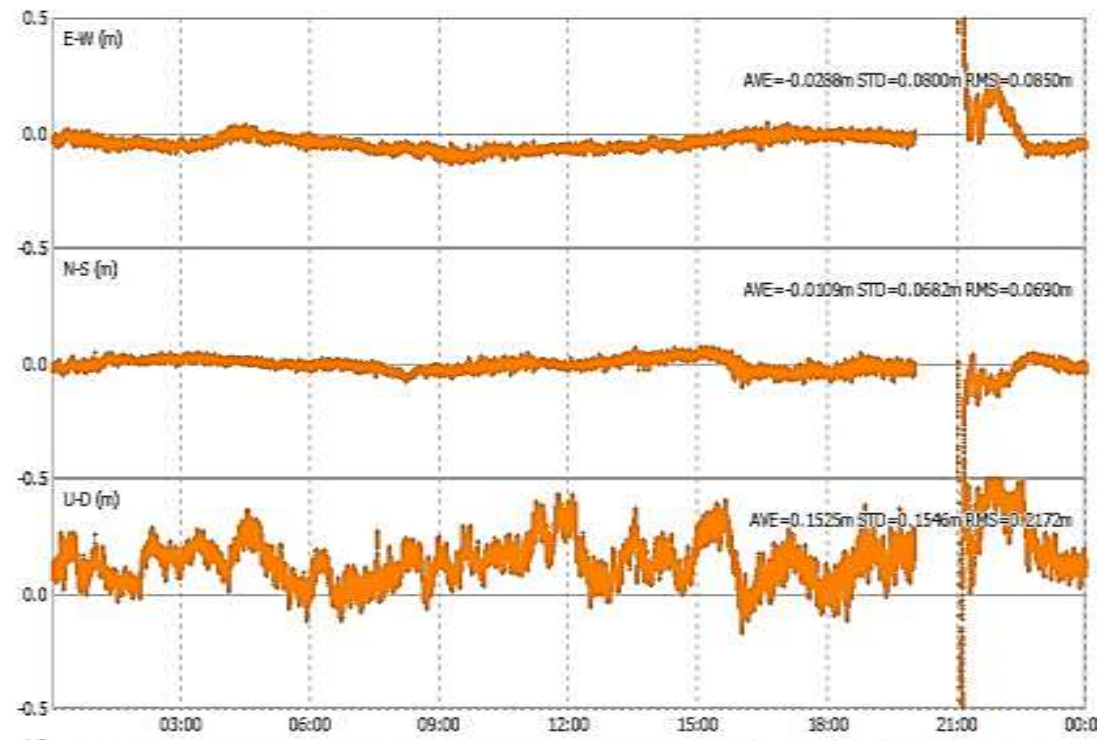

(b)
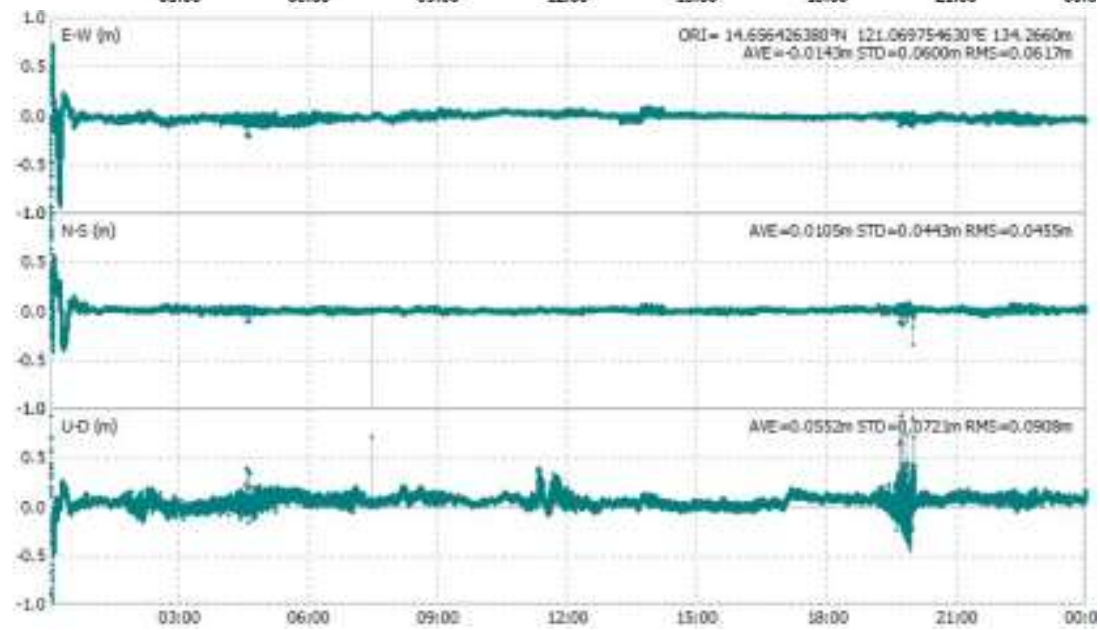

Figure 9: (a) Real-Time solution of MSJ (March 1, 2020) and b) Post-Processed solution of MSJ (March 1, 2020)

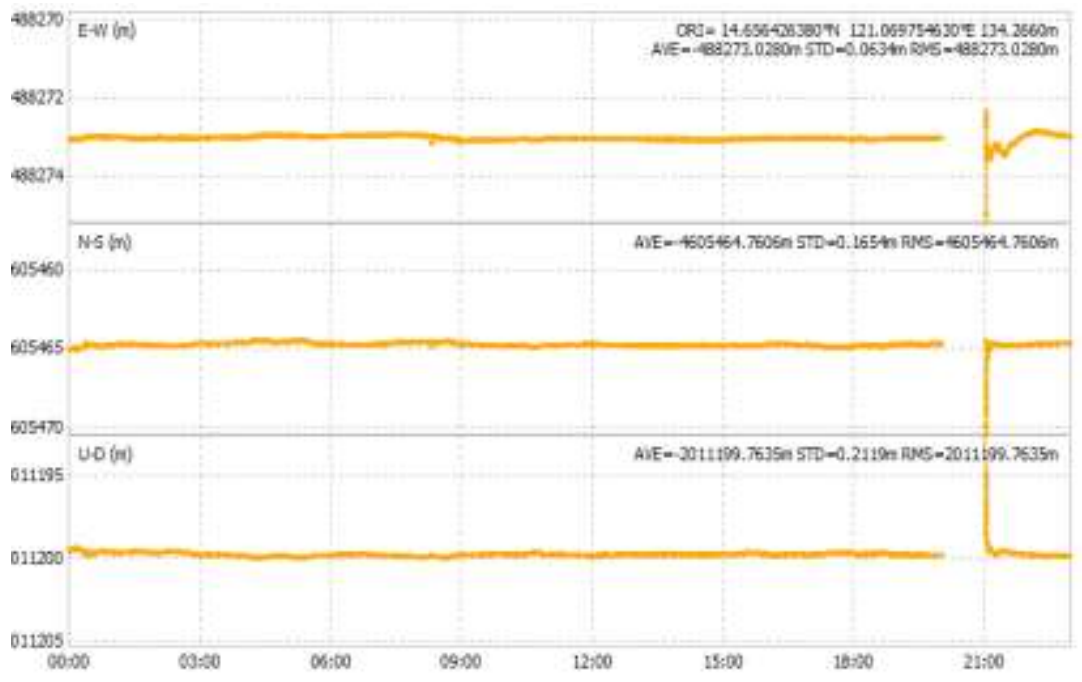

Figure 10: Real-Time solution of MSJ in Curtin University, Australia (March 1, 2020).

Figure 11 shows the comparison of the solution plot of Trimble NetR9 (blue line) and MSJ-3008-GM4-QZS (orange and red lines) using RTKPLOT. Trimble NetR9 gave a PPP solution but it takes some time to converge before achieving centimeter level accuracy. The orange line is the real-time plot of the MSJ-3008-GM4-QZS solution while 
the red line is the anomalous single point solution around 20:00 to 21:00 UTC from the same receiver. After said period, the real time solution took 30 to 60 minutes before converging again. As mentioned, this anomaly needs further verification.

Using RTKPLOT, the statistics for each solution plot is recorded. The root mean square for the whole month of March is presented in Table 4. In Figures 12, 13, and 14, the daily statistical values for horizontal direction is plotted. It is observed that there are days when MSJ-3008-GM4-QZS solution has smaller average error than NetR9 and vice versa which can be considered to varying daily conditions. The deviation for both solutions is all below $10 \mathrm{~cm}$, which means that a real-time PPP solution can achieve centimeter error with respect to the true position of the base station receiver. It should be noted that the initialization after the period with no solution is included in the computation of statistics. This is evident in the constant difference in the precision and accuracy between the two receivers' standard deviation and root mean square plots. The two outlier points on March 6 and 26, 2020 are due to noises and jumps during re-convergence. Figures 15, 16, and 17 present the absolute average error, standard deviation, and root mean square in the vertical direction.

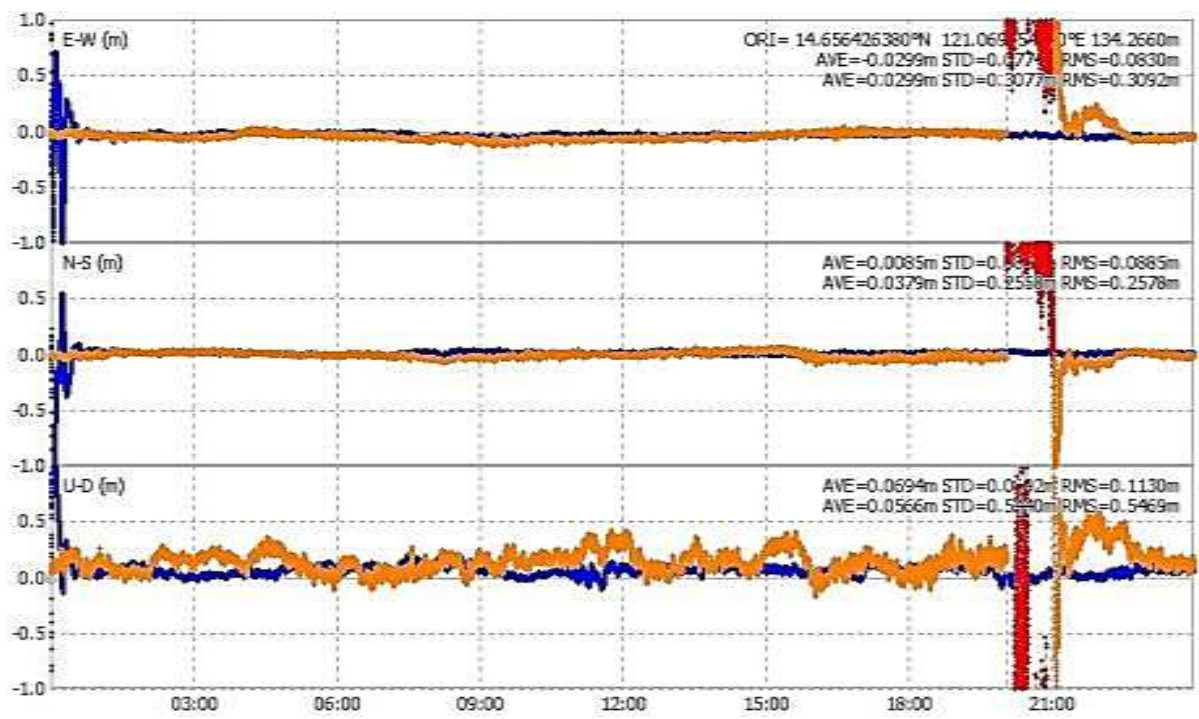

Figure 11: NetR9 (Blue) and MSJ Real-time (Orange and Red) (March 1, 2020)

Table 4: MSJ-3008-GM4-QZS and Trimble NetR9 accuracy

\begin{tabular}{ccc} 
& MSJ-3008-GM4-QZS & NetR9 \\
\hline RMS (cm) & RMS (cm) \\
N-S & 10.45 & 6.27 \\
U-D & 6.27 & 2.32 \\
\end{tabular}

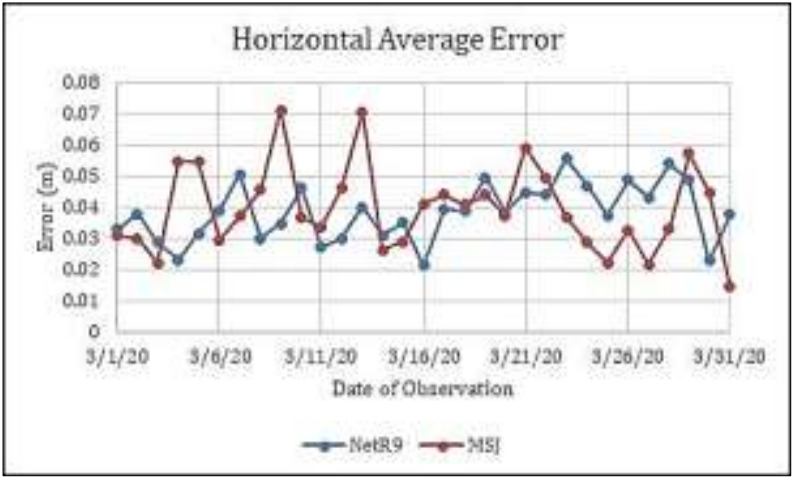

Figure 12: Horizontal Average Error

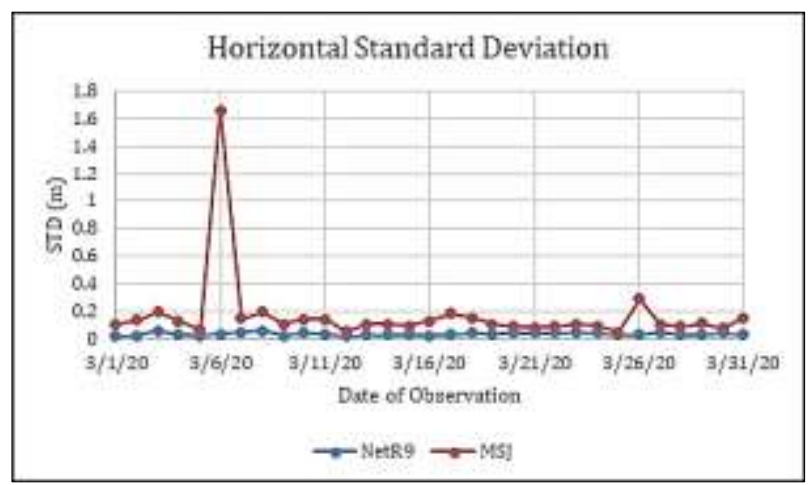

Figure 13: Horizontal Standard Deviation 


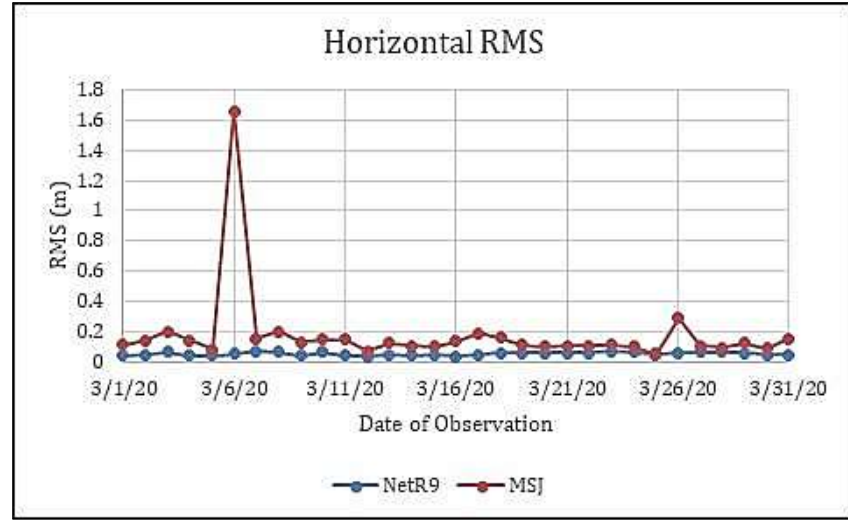

Figure 14: Horizontal Root Mean Square

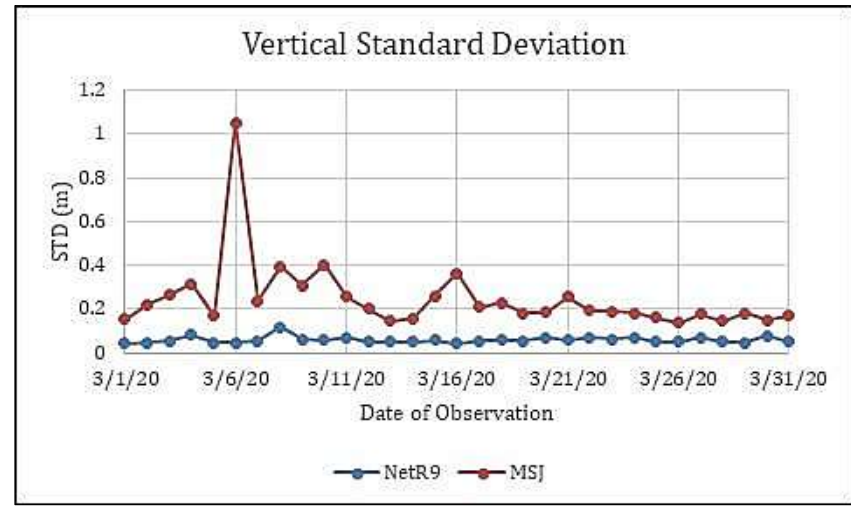

Figure 16: Vertical Standard Deviation

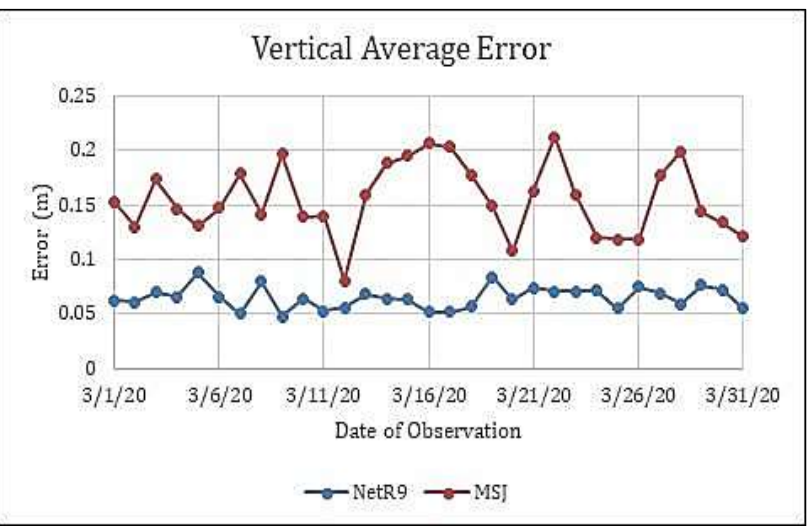

Figure 15: Vertical Average Error

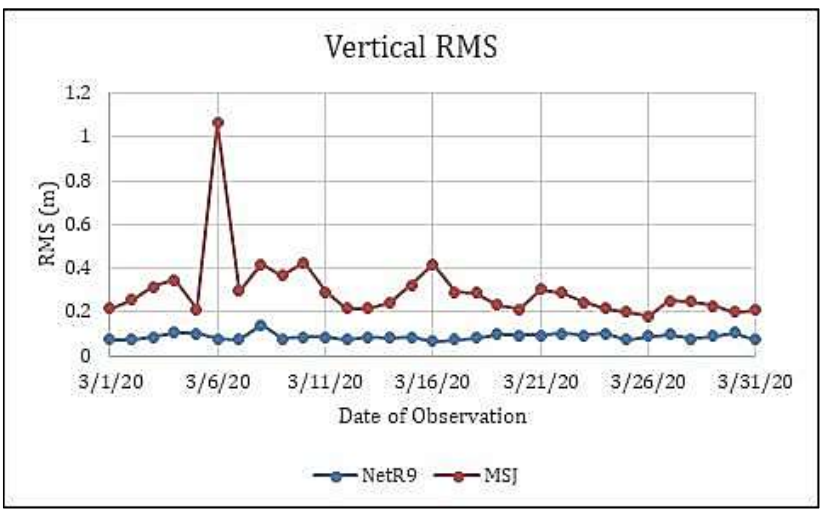

Figure 17: Vertical Root Mean Square

Taking out the solutions after the 21:00 UTC where large errors were observed prior to re-convergence, the performance of the MSJ-3008-GM4-QZS improved to around $7.17 \mathrm{~cm}, 3.58 \mathrm{~cm}$, and $22.32 \mathrm{~cm}$ in the East, North and Up directions, respectively.

\section{CONCLUSIONS AND RECOMMENDATIONS}

Converging time and accuracy were the parameters used to evaluate the performance of the receivers in PPP solutions using MADOCA. Based on the results, the average convergence time of ZED-F9P is 26 minutes and 33 seconds, and Trimble NetR9 had 18 minutes and 28 seconds. A difference of about 8 minutes and 5 seconds. The statistical values showed that ZED-F9P achieved sub-decimeter accuracy on 9 out of 10 days of complete 24-hour observations. The accuracy was computed at $5.28 \mathrm{~cm}, 2.89 \mathrm{~cm}$, and $9.55 \mathrm{~cm}$ RMS on the East, North, and Up directions, respectively. These results are comparable with the Trimble NetR9 solution with $3.54 \mathrm{~cm}, 2.55 \mathrm{~cm}$ and $8.74 \mathrm{~cm}$.

The assessment of QZSS satellites availability showed that these satellites are visible throughout the day and can broadcast LEX signal with acceptable signal quality. This means that MADOCA-PPP can be used in the Philippines. The evaluation of MSJ-3008-GM4-QZS showed RMSE of $10.45 \mathrm{~cm}, 6.27 \mathrm{~cm}$, and 27.56 in the East, North and Up directions, respectively. Its accuracy was degraded due to large errors after the anomalous observations between the period 20:00 to 21:00 UTC. Cutting the solutions from 21:00 UTC onward, the receiver was able to achieve $7.17 \mathrm{~cm}$, $3.58 \mathrm{~cm}$, and $22.32 \mathrm{~cm}$ in the East, North and Up directions, respectively.

\section{ACKNOWLEDGEMENT}

Thank you to Tokyo University of Marine Science and Technology (TUMSAT) for the receivers and assistance to this study. 


\section{REFERENCES}

[1] QZSS. eoPortal Directory. 2020. https://directory.eoportal.org/web/eoportal/satellitemissions/q/qzss\#: : text=QZSS\%20signals\%3A\&text=There\%20are\%20L1C\%2C\%20L1\%2DC,signal $\% 20$ on $\%$ 20the\%20E6\%2Dband.

[2] Wang, G.; Kuang, C.; Cai, C.; Yi, Z.; Dai, W. Real-time PPP based on the Quasi-Zenith Satellite System MADOCA-LEX signal. In IET Radar, Sonar \& Navigation, vol. 12, no. 5, pp. 494-498, 2018.

[3] Yamada, H.; Fujita, S.; Saitoh, S.; Sakai, T.; Real-time simulation of QZSS regional satellite navigation. Proc Int. Conf. Institute of Navigation, vol 137, no. 1, pp. 323-330. September 2012.

[4] Zhu, S.; Yue, D.; He, L.; Liu, Z.; Chen, J. Comprehensive analysis of compatibility between QZSS and GPS in Asia-Pacific region: Signal quality, RTK and PPP. Advances in Space Research, Volume 66, Issue 2, pp. 395 411. 2020.

[5] Saito, M.; Sato, Y.; Miya, M.; Shima, M.; Omura, Y.; Takiguchi, J.; Asari, K. Centimeter-class augmentation system utilizing quasi-zenith satellite. Proc. Int. Conf. Institute of Navigation, Portland, OR, pp. 1243-1253, September 2011.

[6] Kishimoto, M.; Myojin, E.; Kogure, S.; Noda, H.; Terada, K. QZSS On Orbit Technical Verification Results. Proc. Int. Conf. Institute of Navigation, Portland, OR, pp. 1206-1211, September 2011.

[7] Overview of the Quasi-Zenith Satellite System. 2020. https://qzss.go.jp/en/overview/services/sv01_what.html

[8] Choy, S.; Harima, K.; Li, Y.; Choudhury, M.; Rizos, C.; Wakabayashi, Y.; Kogure, S. GPS Precise Point Positioning with the Japanese Quasi-Zenith Satellite System LEX Augmentation Corrections. Journal of Navigation, Vol 68, pp. 1-15. 2015.

[9] Vietsel, A.; Lebedinsky, A.; Nikitin, D.; Positioning Technology with QZSS signals. Proc. Int. Conf. Institute of Navigation, San Diego, CA, pp. 441-449. January 2014.

[10]Duong, V.; Harima, K.; Choy, S.; Rizos, C. Performance of precise point positioning using current triplefrequency GPS measurements in Australia. 2016.

[11] Harima, K.; Choy, S.; Rizos, C.; Kogure, S. An investigation into the performance of real-time GPS+GLONASS Precise Point Positioning (PPP) in New Zealand, Journal of Applied Geodesy, 11(3), pp. 185-195. 2017.

[12] Reyes, R.; Tihoshi, A.; Franco, J.; Colegio, C.; Rada, W. Performance Analysis of Single Point Positioning (SPP) and MADOCA-Precise Point Positioning (MADOCA-PPP) in Road/Lane Identification. Asian Journal of Engineering and Technology, 5(6). 2017.

[13] Hoang-Van, H.; Thanh, T.; The, V., "An evaluation of precise point positioning using QZSS LEX signal in Vietnam", NICS 2016 - Proceedings of 2016 3rd National Foundation for Science and Technology Development Conference on Information and Computer Science, pp.234-239, 2016.

[14] Kanzaki, M.; Noguchi, N. Experimental Results of LEX Corrections Using Farming Machine, Sixth Meeting of the International Committee on Global Navigation Satellite Systems (ICG), Tokyo, Japan, September 2011.

[15] Suzuki, T.; Kubo, N. Precise point positioning for mobile robots using software GNSS receiver and QZSS LEX signal. IEEE/RSJ International Conference on Intelligent Robots and Systems, pp. 369-375. 2013.

[16] Gill, M.; Bisnath, S.; Aggrey, J.; Seepersad, G. Precise Point Positioning (PPP) using Low-Cost and Ultra-LowCost GNSS Receivers. Institute of Navigation (ION GNSS+ 2017). 2018.

[17] Realini, E.; Caldera, S.; Pertusini, L.; Sampietro, D. Precise GNSS Positioning Using Smart Devices. Sensors (Basel, Switzerland), 17(10), 2434. 2017.

[18] Nie, Z.; Liu, F.; Gao, Y. Real-time precise point positioning with a low-cost dual-frequency GNSS device. GPS Solutions 24, 9. 2020.

[19]Duong, V.; Harima, K.; Choy, S.; Rizos, C. Performance of precise point positioning using current triplefrequency GPS measurements in Australia. 2016.

[20] Suzuki, T.; Kubo, N.; Takasu, T.,’Evaluation of precise point positioning using MADOCA-LEX via Quasi-Zenith satellite system", Institute of Navigation International Technical Meeting, 2014.

[21] List of products that support Quasi-Zenith Satellite System. QZSS. 2020. https://qzss.go.jp/en/usage/products/list.html

[22] MADOCA-PPP. GNSS Tutor. http://www.denshi.e.kaiyodai.ac.jp/gnss tutor/madoca.html

[23] MADOCA Real-Time Products. QZSS. https://ssl.tksc.jaxa.jp/madoca/public/public_index en.html

[24] Ublox ZED-F9P module, 2019. https://www.u-blox.com/en/product/zed-f9p-module.

[25] Multi-frequency Multi-GNSS Solution Overview, Magellan Systems Japan, Inc., https://www.magellan.jp/english/item/index6.html

[26] MADOCA-SSR. ftp://mgmds01.tksc.jaxa.jp/

[27] Takasu, T.; Kubo, N.; Yasuda, A., "Development, evaluation and application of RTKLIB: a program library for RTK-GPS”, GPS/GNSS Symposium, 2007. 